International Journal of Case Reports
(ISSN:2572-8776)

\title{
Non-resolving acute sinusitis due to nasal hamartoma: a case report
}

\section{Fausto Martinez, $\mathrm{MD}^{1,2}$, David Zambrano, $\mathrm{MD}^{1}$}

${ }^{1}$ Department of Otolaryngology - Head and Neck Surgery, Rhinology \& Skull Base Surgery, Hospital Vozandes Quito, Ecuador; ${ }^{2}$ Professor of Otolaryngology, Pontificia Universidad Católica del Ecuador

\section{ABSTRACT}

The paranasal sinuses are closely related to vital structures, and therefore, rhinosinusitis may lead to various dangerous complications. Nasal chondromesenchymal hamartoma is a rare benign lesion of the sinonasal tract in children and adolescents. We report the case of a 15-year-old patient with antibiotic-refractory acute sinusitis in whom a nasal tumor was identified and histopathologically diagnosed as a hamartoma. Therefore, the tumor was removed surgically to alleviate the patient's symptoms. This *Correspondence to Author: Fausto Martinez, MD ${ }^{1,2}$

${ }^{1}$ Department of Otolaryngology Head and Neck Surgery, Rhinology \& Skull Base Surgery, Hospital Vozandes Quito, Ecuador; ${ }^{2}$ Professor of Otolaryngology, Pontificia case highlights the necessity of investigating the underlying cause if conventional treatment fails to resolve a mild disease like acute sinusitis. To the best of our knowledge, this is the first reported case of a chondro-osseous respiratory epithelial adenomatoid hamartoma presenting with acute sinusitis.

Universidad Católica del Ecuador

How to cite this article:

Fausto Martinez, David Zambrano. Non-resolving acute sinusitis due to nasal hamartoma: a case report. International Journal of Case Re-

Keywords: nasal hamartoma, acute sinusitis, nasal endoscopy ports, 2021, 5:233.

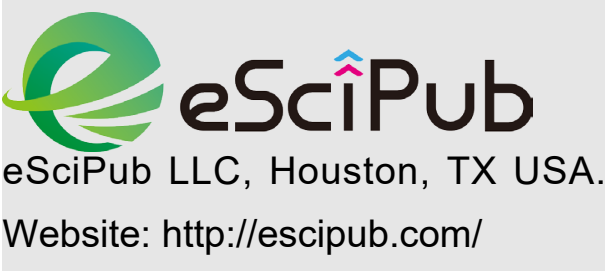




\section{INTRODUCTION:}

Acute sinusitis can present with symptoms that are related to the affected paranasal sinuses. These include frontal headache, fever, and fatigue; and may be accompanied by tenderness over the medial part of the infraorbital margin. Due to its close relation to many vital structures, rhinosinusitis may give rise to various dangerous complications ${ }^{[1]}$.

Patients whose condition worsens clinically or fails to improve after 3-5 days of empiric antimicrobial therapy with a first-line agent should be evaluated for the possibility of having resistant pathogens, a non-infectious etiology, structural abnormality, or other causes of treatment failure ${ }^{[2]}$.

Nasal chondromesenchymal hamartoma is a rare benign lesion of the sinonasal tract in children and adolescents. Commonly, it appears as a nasal obstruction, an intranasal mass or facial swelling in young infants and children, and rarely in adults. Usually, it is benign and can be cured by complete excision ${ }^{[3]}$. Here, to the best of our knowledge, we report the first case of a patient with antibiotic-refractory acute sinusitis in whom a nasal tumor was histopathologically diagnosed as a chondro-osseous respiratory epithelial adenomatoid hamartoma (COREAH).

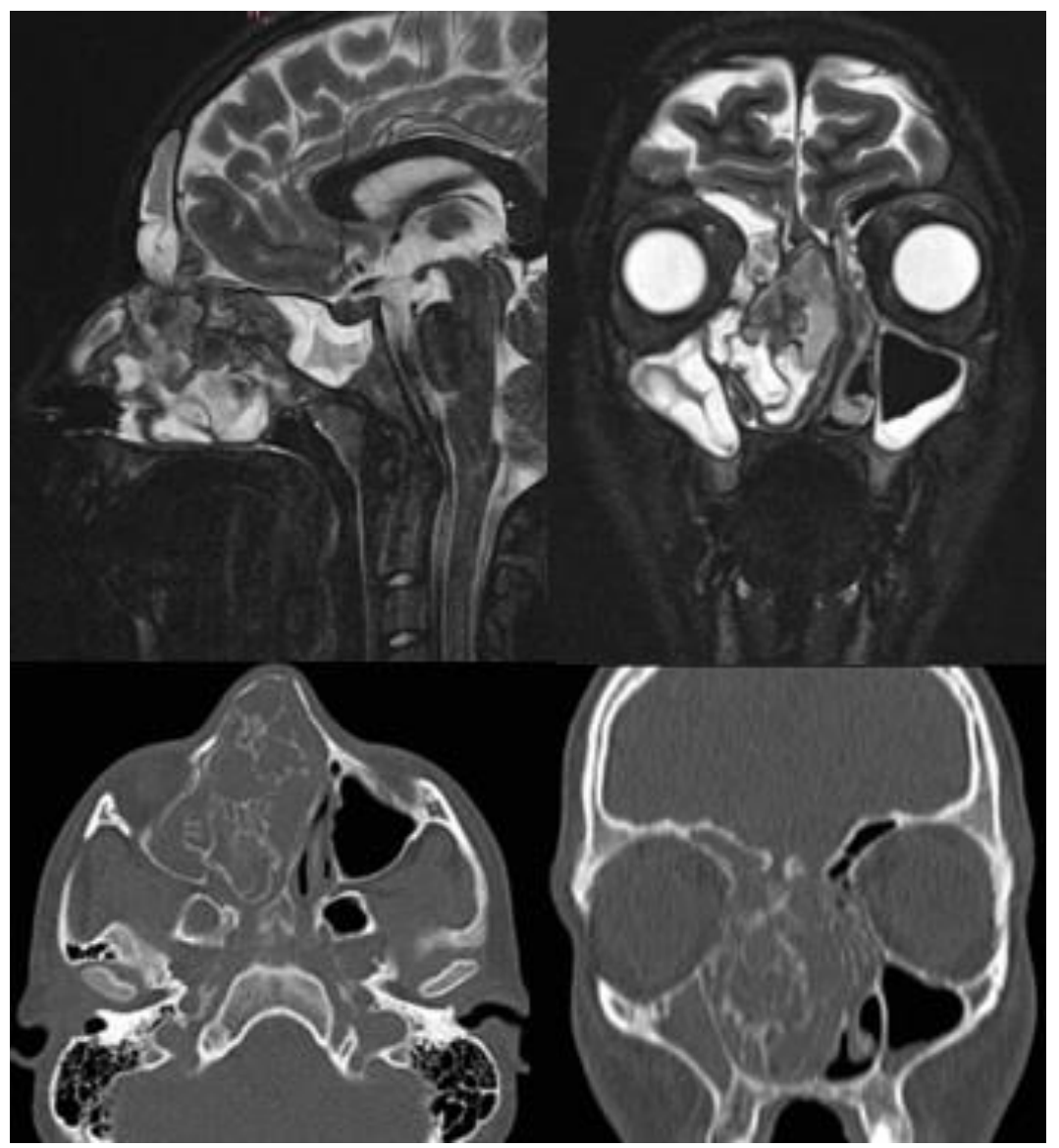

Figure 1: Computed tomography (CT) and magnetic resonance imaging (MRI) prior to surgery.

\section{CSE PRESENTATION}

A 15-year-old patient, without any important medical antecedents, visited the emergency department with facial pain and frontal lobe headache that radiated to the right retro-orbital region. Bilateral nasal obstruction was present, and rhinorrhea had evolved to mucopurulent discharge in two weeks. The patient had received oral antibiotic treatment with cefuroxime without amelioration of the symptoms. 
On physical examination, fever and a broadened nasal bridge were observed. Eyelid edema was not present, and ocular movements were preserved. There were no signs of meningitis. On nasal endoscopy, a pink and irregularly shaped mass totally obstructing the right nostril and left septal deviation were found. Laboratory tests reported elevated white blood cell count and C-reactive protein level.

Computed tomography (CT) and magnetic resonance imaging (MRI) confirmed an expansive mass in the right nostril with osseous trabeculae and zones with a soft tissue density between 24 and $44 \mathrm{UH}$. Near the right maxillary, frontal and sphenoid sinuses, soft tissue opacity with a density of $28 \mathrm{UH}$ was also observed (Figure 1).

The patient was admitted for in-patient management. Antibiotic therapy with ampicillinsulbactam, dexamethasone and analgesia were administered. In addition, a biopsy was taken via nasal endoscopy. The patient was discharged after 48 hours when his condition improved, and was asked to follow-up after the histopathology report became available.

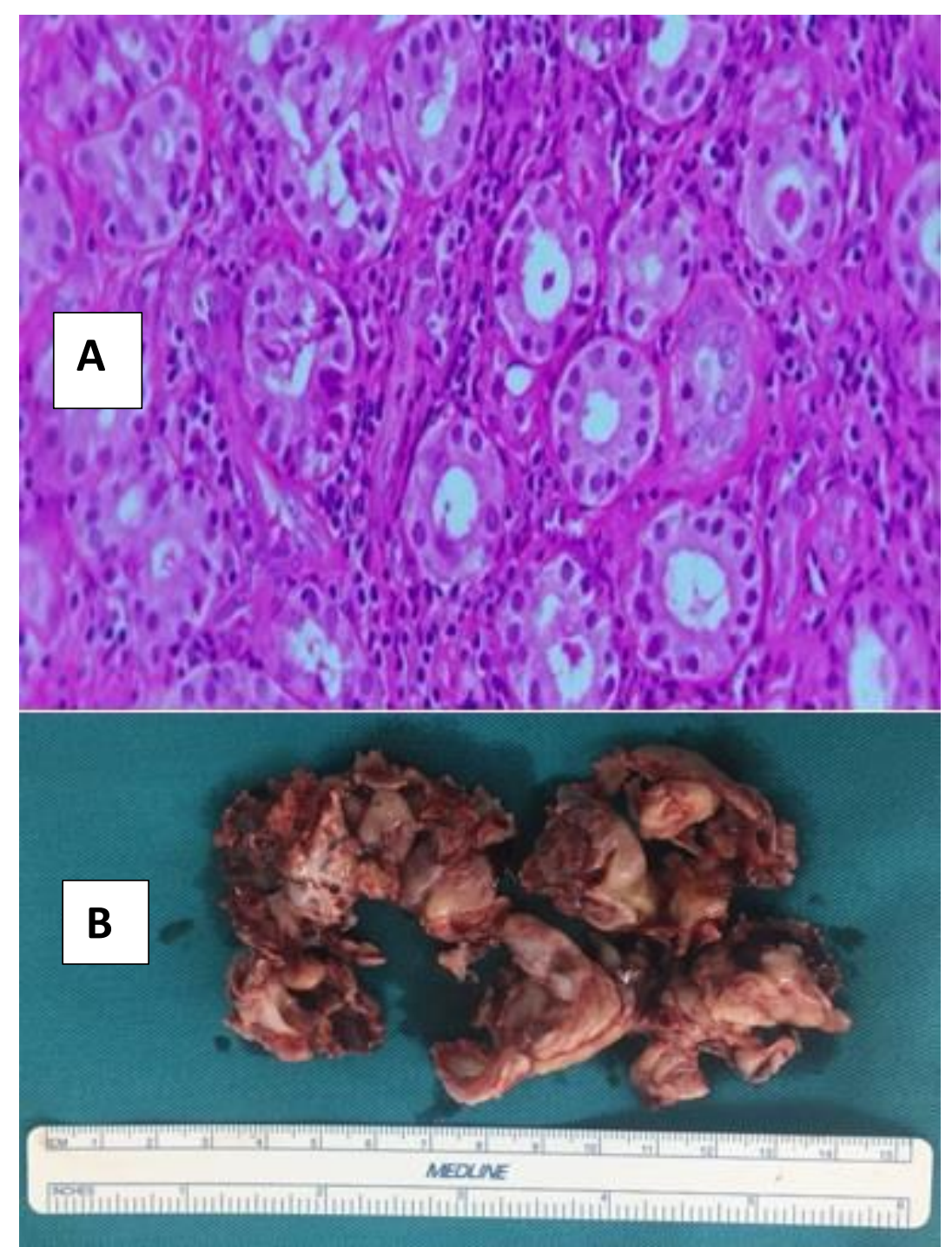

Figure 2: A, The pathological report indicated the presence of an adenoepithelial respiratory hamartoma with chondro-osseous components. B, Macroscopically complete excision of the tumor (Figure 2).

The patient returned to the emergency headache, right retro-ocular pain, and fever of department after 7 days with an intense frontal $39^{\circ} \mathrm{C}$. The pathological report indicated the IJCR: https://escipub.com/international-journal-of-case-reports/ 
presence of an adenoepithelial respiratory hamartoma with chondro-osseous components (Figure 2).

Endoscopic resection of the lesion was performed and completed using the Draf Ilb procedure. We were able to perform a macroscopically complete excision of the tumor (Figure 2). The patient was discharged 48 hours after the procedure with a prescription of antibiotic therapy. He did not have any orbital or intracranial complications. He was regularly followed-up for 2 years and there was no recurrence of the lesion (Figure 3).

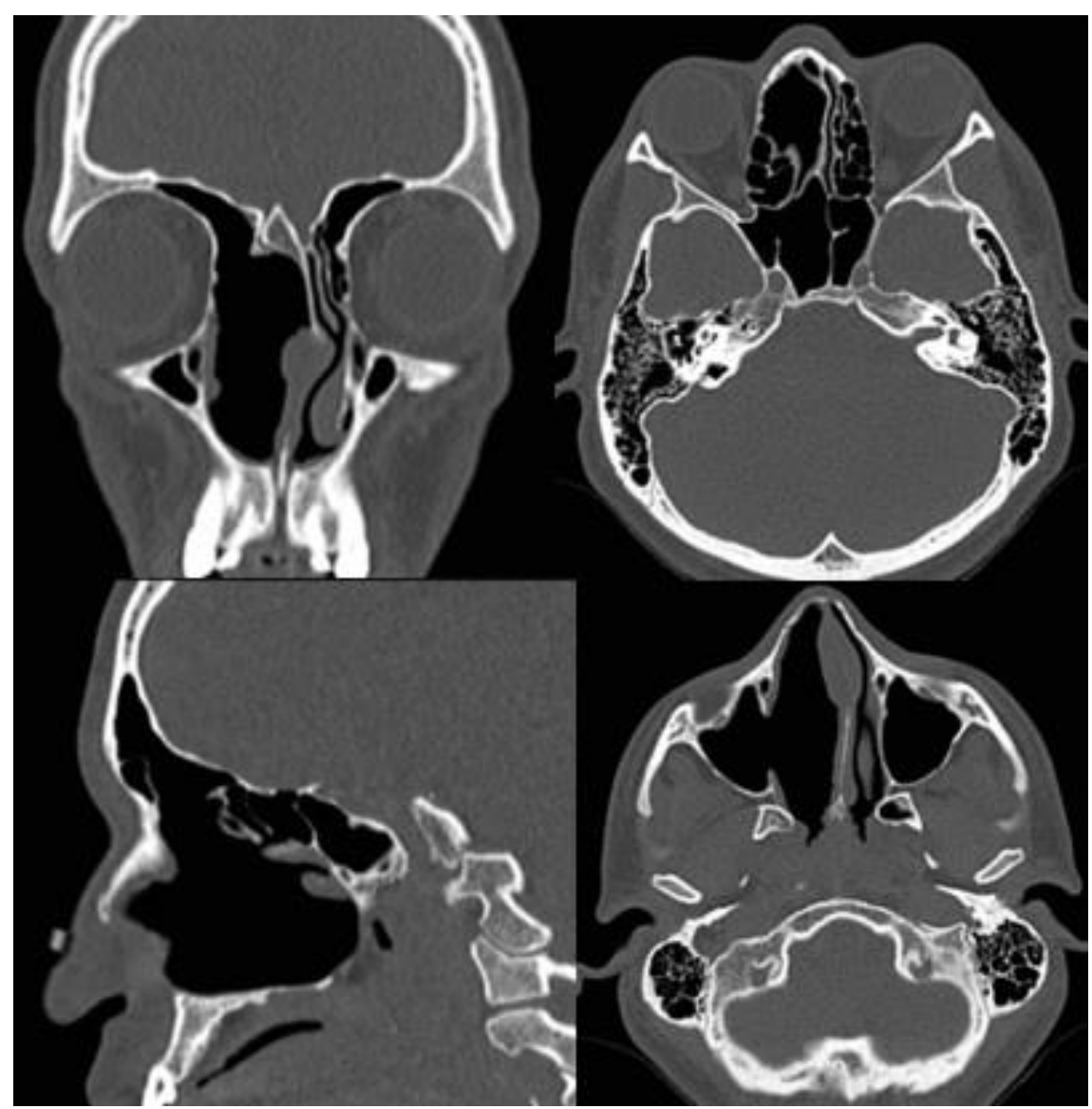

Figure 3: Postoperative Computed tomography (CT)

DISCUSSION: COREAHs are rare and unique lesions, which may belong to a spectrum of hamartomatous lesions, extending from purely epithelial hamartomas at one end to purely mesenchymal hamartomas at the other ${ }^{[4]}$.

COREAHs are different from other types of hamartomas and have not shown a predisposition to a particular age group or sex. Therefore, cases of 3- to 59-year-old patients belonging to both sexes have been reported ${ }^{[5]}$.

Patients with COREAHs present with nonspecific symptoms, such as nasal obstruction, nasal discharge, facial pain, and olfactory impairment or loss.
Less frequent symptoms include proptosis, enophtalmos or impaired eye movement, intracranial invasion, and osseous erosion [4].

To the best of our knowledge, this is the first reported case where a patient with COREAH presented with acute sinusitis.

The endoscopic examination showed a unilateral, hard, pink, cerebriform lesion that had displaced the nasal septum and was associated with purulent rhinorrhea.

CT and MRI confirmed the presence of a large mass. These modalities did not show the characteristic findings because they vary 
according to the main element of the hamartoma.

Nevertheless, COREAHs can be suspected if an expansive mass of soft tissue with or without calcifications but without osseous erosion or intracranial extension is present. MRI shows a hypo/isointense mass on $\mathrm{T} 1$ and a cerebriform pattern on T2-weighted images ${ }^{[5]}$.

In this case, the tumor originated from the ethmoidal cells. Of the very few reported cases so far, the tumor originated from the nasal cavity in the majority whereas it originated from the ethmoidal cells in only one case ${ }^{[4]}$.

Nasal polyps, inverted papilloma, nasoethmoidal encephalocele, nasal glioma, rhabdomyosarcomas, lymphomas, and chondrosarcomas can be considered as the differential diagnoses.

The chosen treatment was complete surgical resection. For this, an endoscopic approach was carried out with a maxillary antrostomy, anterior and posterior ethmoidectomy completed using the Draf Ilb procedure. Complete resection of the lesion was achieved, which was curative in all the cases reported in literature. Herein, once the lesion was removed, the infectious process was controlled with antibiotic therapy. Recurrence was reported in one case which probably occurred due to an initial incomplete resection ${ }^{[4]}$.

It is important to identify these lesions preoperatively. Otherwise, the procedure may result in either aggressive or incomplete resection [5].

\section{CONCLUSION}

COREAHs are benign sinonasal lesions that are frequently diagnosed incidentally. They should be considered as a differential diagnosis in all age groups to avoid unnecessary or aggressive treatment. Owing to this, in cases of complicated acute sinusitis that do not respond to adequate conventional treatment, it is necessary to investigate a non-infectious etiology such as structural abnormalities.

\section{CONSENT:}

The patient provided written informed consent.

\section{CONFLICTS OF INTEREST:}

The authors declare that there is no conflict of interest.

\section{FUNDING:}

This research did not receive any specific grant from funding agencies in the public, commercial, or not-for-profit sectors.

\section{ETHICAL STATEMENT:}

The patient provided written informed consent at Hospital Vozandes Quito regarding the use of his health information for the purposes of research.

\section{ACKNOWLEDGEMENTS:}

We would like to thank Eng. Maria C. Nevarez Martinez, MSc for their support in conducting this research

\section{REFERENCES:}

[1] Al Yaeesh I, AlOmairin A, Al Shakhs A, Almomen A, Almomen Z, AlBahr A, et al. The serious complications of frontal sinusitis, a case series and literature review. J Surg Case Rep 2020;2020:rjaa474.

https://doi.org/10.1093/jscr/rjaa474.

[2] Chow AW, Benninger MS, Brook I, Brozek JL, Goldstein EJC, Hicks LA, et al. Executive summary: IDSA clinical practice guideline for acute bacterial rhinosinusitis in children and adults. Clin Infect Dis 2012;54:1041-5. https://doi.org/10.1093/cid/cir1043.

[3] Vijayasundaram S, Gopalakrishnan S, Karthikeyan P, Vignesh R. Nasal chondromesenchymal hamartoma: a rare benign lesion in adult female. Indian J Otolaryngol Head Neck Surg 2021:1-3. https://doi.org/10 .1007/s12070-020-02333-7.

[4] Fang G, Wang C, Piao Y, Zhang L. Chondroosseous respiratory epithelial adenomatoid hamartoma of the nasal cavity. Pediatr Int 2016;58:229-231.

https://doi.org/10.1111/ped.12777.

[5] Villarreal IM, Pinilla M, Salas I, Garcia Y, LópezCortijo C. Respiratory epithelial adenomatoid hamartoma: A very rare entity originating from the lateral nasal wall. Eur Ann Otorhinolaryngol Head Neck Dis 2015;132:369-70. https://doi.org/10.101 6/j.anorl.2015.08.003.

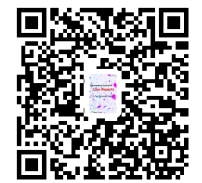

IJCR: https://escipub.com/international-journal-of-case-reports/ 